\title{
Early Withdrawal of Axons from Higher Centers in Response to Peripheral Somatosensory Denervation
}

\author{
Alessandro Graziano and Edward G. Jones \\ Center for Neuroscience, University of California, Davis, Davis, California 95618
}

The mechanisms responsible for long-term, massive reorganization of representational maps in primate somatosensory cortex after deafferentation are poorly understood. Sprouting of cortical axons cannot account for the extent of reorganization, and withdrawal of axons of deafferented brainstem and thalamic neurons, permitting expression of previously silent synapses, has not been directly demonstrated. This study is focused on the second of these. In monkeys, deafferented for two years by section of the cuneate fasciculus at the $\mathrm{C} 1$ level, there was extensive withdrawal of axon terminals from thalamus and cortex, detectable a decade before visible atrophy of their parent neuronal somata in the cuneate nucleus or thalamus. Slow, inexorable progression of lemniscal and thalamocortical axonal withdrawal is a neurodegenerative phenomenon likely to be a powerful inducement to compensatory long-term plasticity, a mechanism that can explain the long-term evolution of cortical reorganization and, with it, phantom sensations in spinal patients and amputees.

\section{Introduction}

The primate somatosensory cortex is remarkable for its capacity to reorganize in the face of perturbations of peripheral input. Adaptations can be positive, such as enlargement of a representation of digits engaged in repetitive actions (Recanzone et al., 1992; Pantev et al., 2001), or negative, such as central pain after amputation of a limb or peripheral nerve injury (Flor et al., 1995; Moore et al., 2000). The most evident cortical response to deafferentation is expansion of representations of body parts with intact peripheral innervation at the expense of those deprived of sensory input (Kaas and Florence, 2001).

Short-term plasticity of somatosensory cortical and subcortical neurons after limited denervation depends on uncovering of silent synapses, release of GABA-mediated inhibition and modulation of synaptic efficacy (Wall, 1977; Buonomano and Merzenich, 1998; Calford, 2002; Cowan et al., 2003). Mechanisms underlying long-term plasticity, especially the massive expansion of a representation with intact innervation occurring after extensive deafferentation of an adjoining body part (Pons et al., 1991; Jones and Pons, 1998), are less well understood.

Hypotheses about the basis for massive long-term expansions of cortical maps depend either on sprouting of axons of non-deafferented central neurons and formation of synapses on deafferented neurons (Kaas et al., 2007), or on withdrawal

\footnotetext{
Received Nov. 6, 2008; revised Jan. 26, 2009; accepted Feb. 14, 2009.

This work was supported by Grant NS 21377 from the National Institutes of Health, United States Public Health Service, and by the University of California, Davis, W. M. Keck Program in Cellular and Molecular Neuroscience Imaging. We thank Phong L. Nguyen for technical help.

Correspondence should be addressed to Dr. Edward G. Jones, 1544 Newton Court, Davis, CA 95618. E-mail: ejones@ucdavis.edu.

DOI:10.1523/JNEUROSCI.5388-08.2009

Copyright $\odot 2009$ Society for Neuroscience $\quad 0270-6474 / 09 / 293738-11 \$ 15.00 / 0$
}

of overlapping inputs from intact neurons to reveal hitherto silent inputs from body parts with intact innervation (Jones, 2000). The demonstrated extent of axon sprouting cannot account for the slow evolution of long-term plasticity and cannot explain the wide extent of cortical reorganization (Jones, 2000). The withdrawal hypothesis depends on the fact that overlap in the thalamic input to cortex is more extensive than representations revealed by extracellular microelectrode mapping (Jones, 2000). As a consequence of extensive divergence of ascending axons at all levels of the somatosensory system (Jones et al., 1997; Rausell et al., 1998), subthreshold input from a single digit normally extends over many millimeters of cortex, and $>30 \%$ of the thalamic representation of the hand can be destroyed before the cortical representation of a digit begins to shrink (Jones et al., 1997).

The withdrawal hypothesis, although not experimentally verified, was supported by the fact that neurons along the path from periphery through brainstem and thalamus to somatosensory cortex do not remain static in the face of extensive loss of peripheral input by nerve or spinal lesions. They undergo a slow, progressive atrophy that spreads transneuronally across peripheral synapses in the dorsal column nuclei and across lemniscal synapses in the thalamus, manifested by shrinkage and eventually death of the relay neurons (Woods et al., 2000). The extent to which this transneuronal atrophy of deafferented neurons in the dorsal column nuclei and thalamus is accompanied by withdrawal of their axons from thalamus and cortex, respectively, and especially how soon it commences after deafferentation, was unknown. The present study shows that axonal withdrawal from these higher centers occurs long before the appearance of detectable atrophy in the deafferented parent neurons. This is likely to represent an important impetus to compensatory adjustments of representational maps in the cortex. 

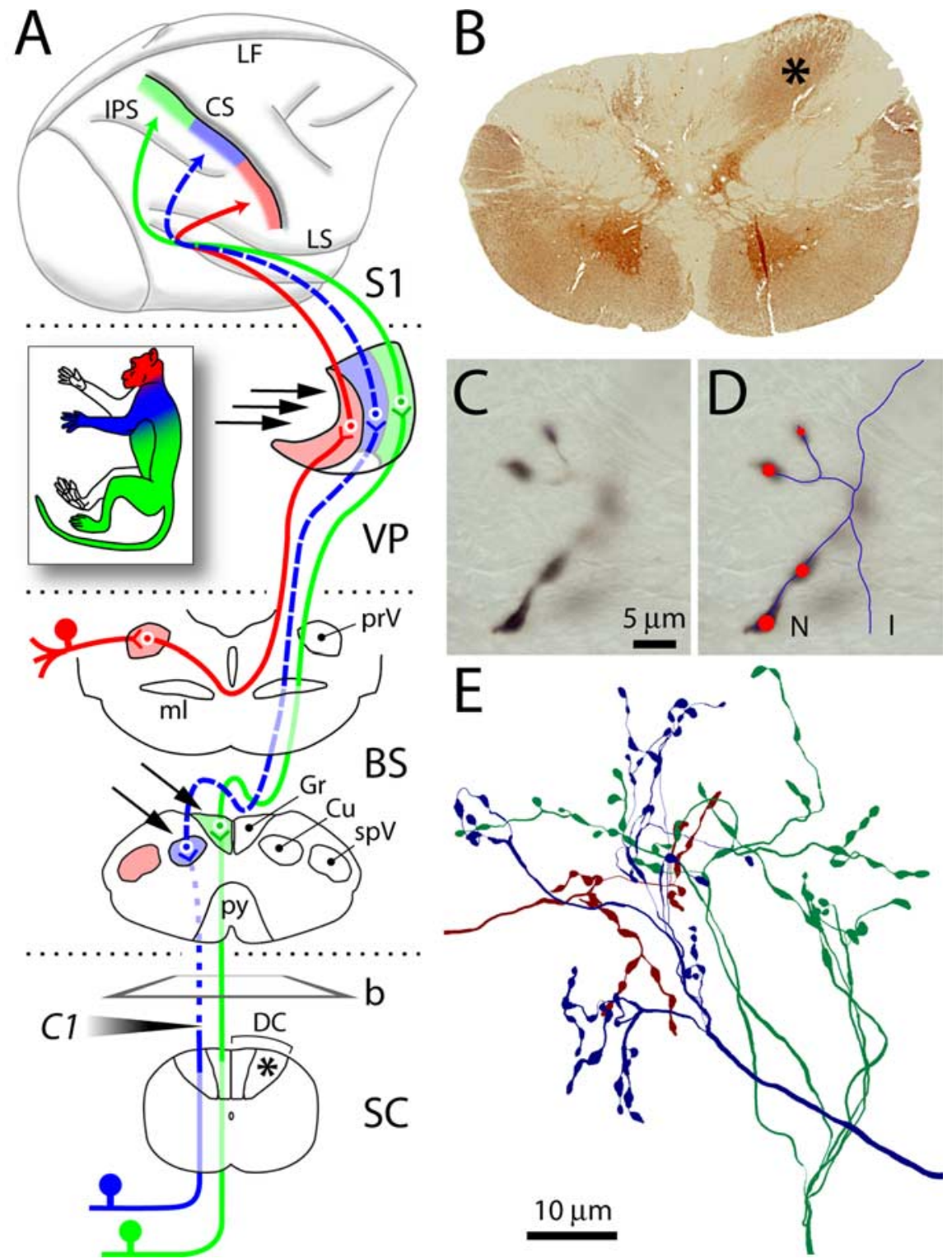

Figure 1. Dorsal column lesion and tract tracing. $\boldsymbol{A}$, The lemniscal and trigeminal pathways convey topographically organized somatosensory information (color coded as in inset) from large-diameter primary sensory axons on the left side of the body to the right thalamus (VP) and cortex (SI). Lesion of the left cuneate fasciculus at the $(1$ level of the spinal cord (SC) (shaded arrowhead) caused Wallerian degeneration of primary sensory axons above the lesion (dotted line). Dashed lines show the component of the pathway deafferented by the lesion. Arrows point to dorsal column nuclei and VP sites where BDA was injected. b, Plane of cut of the section shown in $\boldsymbol{B}$; asterisks denote normal cuneate fasciculus. $\boldsymbol{B}$, Transverse section from SC immunostained for parvalbumin, showing loss of axons in the left cuneate fasciculus, compared with the right (asterisk). C, Anterogradely labeled axon terminals in VP at high magnification. Only the portion in sharp focus is traced at any given time. $\boldsymbol{D}$, Line rendering of Neurolucida tracing of the same axon through the thickness of the section. Red circles mark synaptic boutons (varicosities). Line and marker sizes were continuously updated to match the size of axons and varicosities. $\boldsymbol{E}$, Cluster of three axons terminating in VP, reconstructed from multiple consecutive sections. BS, Brainstem; $C S$, central sulcus; $\mathrm{Cu}$, cuneate nucleus; DC, dorsal columns; $\mathrm{Gr}$, gracile nucleus; I, incomplete ending; IPS, intraparietal sulcus; LF, longitudinal fissure; LS, lateral sulcus; ml, medial lemniscus; $\mathrm{N}$, normal ending; prV, principal trigeminal nucleus; py, pyramidal decussation; spV, spinal trigeminal nucleus.

\section{Materials and Methods}

This study was conducted on Macaca mulatta monkeys in accordance with the United States Public Health Service Policy on Humane Care and Use of Laboratory Animals, the National Institutes of Health's Guide for the Care and Use of Laboratory Animals, and with approval of the Institutional Animal Care and Use Committee.

Cuneate lesion. Ten animals underwent interruption of the left cuneate fasciculus at the $\mathrm{C} 1$ level of the spinal cord. They were sedated with ketamine (15 mg/kg, i.m.), intubated, and anesthesia was maintained with $2 \%$ isoflurane throughout the duration of the surgery. The head was held in a stereotaxic apparatus (David Kopf Instruments) in a flexed position; the lower medulla was exposed via the foramen magnum and the dura mater was incised to allow a clear view of the dorsal columns. A small incision was made on the pial surface of the cuneate fasciculus to allow insertion of microforceps used to squeeze the cuneate fasciculus for $5 \mathrm{~min}$, to ensure interruption of all its axons. The dura was replaced, the muscles and skin sutured in layers, and the animals were allowed to survive two years before tracer was injected.

Tracer injection. For tracer injections in the dorsal column nuclei, the lower medulla was exposed following the same procedure used to lesion the cuneate fasciculus. Evoked multiunit responses to mechanical stimulation of the skin were recorded to determine the location and depth of the gracile and cuneate nuclei, and fiduciary marks were noted to guide the successive injections of tracer. A solution of $10 \%$ biotinylated dextran-amine (BDA) $10,000 \mathrm{kDa}$ (Invitrogen) in $0.1 \mathrm{M}$ phosphate buffer was injected in the gracile and cuneate nuclei with a 10 $\mu \mathrm{l}$ micrometric syringe (Hamilton) through multiple penetrations. At each site, $1-3 \mu \mathrm{l}$ of BDA solution were slowly delivered at multiple depths, the needle being left in place for $3 \mathrm{~min}$ before retracting it to a more superficial position. After the last ejection, the needle was left in place for $5 \mathrm{~min}$ before extraction. For tracer injections in the thalamus, a burr hole was made in the occipital bone, the dura incised, and an electrode was driven horizontally into the thalamus guided by stereotaxic coordinates. Multiunit responses to somatosensory stimuli were recorded. The electrode was advanced in 100 $\mu \mathrm{m}$ increments to cover the ventral posterior (VP) nucleus on the side contralateral to the cuneate fasciculus lesion throughout its anteroposterior extent. An array of electrode penetrations ensured coverage of the dorsoventral and mediolateral extent of VP. The stereotaxic coordinates obtained from the electrophysiological recordings were used to target BDA injections to VP. The tracer was delivered in multiple penetrations using a technique similar to that used for cuneate and gracile nucleus injections, with multiple deliveries along a single penetration.

After the tracer was injected, the opening in the occipital bone was covered with gelfilm, the muscles and skin sutured, and the animal allowed to recover.

The hand/face border in area $3 \mathrm{~b}$ of the primary somatosensory cortex (SI) contralateral to the cuneate lesion was identified electrophysiologically as for the thalamus, with the difference that electrode penetrations were visually guided in the depth of the posterior bank of the central sulcus.

After 3 weeks, the animals were deeply anesthetized with Nembutal and perfused through the ascending aorta with normal saline, followed by $4 \%$ paraformaldehyde in $0.1 \mathrm{~m}$ phosphate buffer, $\mathrm{pH} 7.4$.

Histology. The brains were blocked, the blocks postfixed overnight, infiltrated with $30 \%$ sucrose in $0.1 \mathrm{M}$ phosphate buffer for cryoprotec- 
tion, and subsequently frozen in dry ice. Blocks of the thalamus were sectioned serially at $30 \mu \mathrm{m}$ in the frontal Horsley-Clarke plane on a sliding microtome. Blocks of the cortex were sectioned in a quasi-horizontal plane orthogonal to the central sulcus. Spinal cord and brainstem blocks were sectioned in the transverse plane. All the sections were collected in cold $0.1 \mathrm{M}$ phosphate buffer and processed for BDA visualization.

Histochemistry. The sections were preincubated for $1 \mathrm{~h}$ in $0.1 \mathrm{M}$ phosphate buffer containing $0.03 \% \mathrm{H}_{2} \mathrm{O}_{2}$ to reduce nonspecific peroxidase activity. After preincubation, the sections were rinsed, incubated in avidinperoxidase complex (ABC; Vector Laboratories), rinsed in phosphate buffer, incubated in phosphate buffer containing $0.02 \% 3,3^{\prime}$ diaminobenzidine $\cdot 4 \mathrm{HCl}$ (DAB, Sigma), and $0.03 \%$ hydrogen peroxide. After rinsing, the sections were mounted on glass slides, dried, dehydrated in increasing concentrations of alcohol, cleared in xylene, and coverslipped with DPX mounting medium (BDH).

Immunohistochemistry. Spinal cord sections were preincubated for $1 \mathrm{~h}$ in blocking solution ( $0.1 \mathrm{M}$ phosphate buffer, $0.25 \%$ Triton X-100, and $3 \%$ normal goat serum). After preincubation, the sections were incubated overnight in the same solution containing monoclonal mouse anti-parvalbumin antibody (dilution, 1:3000; Sigma). After rinsing in phosphate buffer, the sections were transferred to the biotin-conjugated secondary IgG solution (1:200 goat anti mouse (Vector Laboratories). The sections were rinsed in phosphate buffer, incubated in avidin-peroxidase complex (ABC; Vector Laboratories), rinsed in phosphate buffer, and incubated in phosphate buffer containing $0.02 \%$ DAB (Sigma) and $0.03 \%$ hydrogen peroxide. After rinsing, the sections were mounted on glass slides, dried, dehydrated in increasing concentrations of alcohol, cleared in xylene, and coverslipped with DPX mountant.

Axon reconstruction. Anterogradely labeled axons in thalamus and cortex were reconstructed with Neurolucida (MBF Bioscience) using a Zeiss Axioscope 100 microscope (Carl Zeiss) equipped with motorized stage controls for the $X, Y$, and $Z$ axes and a color charge-coupled device (CCD) camera. Axon tracing were produced at high magnification through a Zeiss Apochromat $100 \times, 1.4$ numerical aperture, oilimmersion lens. Live images of the magnified specimen were displayed on a PC monitor; a joystick allowed real-time control of stage movements, including focus. Single-axon branches were drawn on the computer monitor using the mouse. Updating the focus continuously allowed the tracing of an axon and its branches through the thickness of the section. Only the portion of an axon in sharp focus was traced at any given time. The thickness of the axon profile was continuously updated using a separate mouse control. On encountering en-passant and terminal boutons, a circular marker was placed (varicosity), with a diameter corresponding to the short axis of the bouton. This allowed controlling for distortions of varicosity diameter resulting from their orientation in three-dimensional space. Section boundaries and blood vessels were drawn and used as fiduciary marks to align contiguous sections. Single axons were traced across multiple sections, until an ending was reached. If no bouton was present in close proximity $(<10 \mu \mathrm{m})$ to the end point of a branch, the ending was defined Incomplete, otherwise it was defined Normal.

Data analysis. Axon tracings were exported into Neuroexplorer (MBF Bioscience) for quantitative analysis. To test for possible effects of transneuronal atrophy, the following variables were calculated for each axon branch: branch length (in $\mu \mathrm{m}$ ), average branch diameter (in $\mu \mathrm{m}$ ), num- ber of varicosities per branch, average varicosity diameter (in $\mu \mathrm{m}$ ), varicosity density (number of varicosities per $\mu \mathrm{m}$ ), median distance between varicosities (MDBV; in $\mu \mathrm{m}$ ), a measure of bouton clustering independent from branch length, and incomplete ending (binary: present/absent).

The following variables were included in the analysis to examine possible consequences of deafferentation, affecting the geometry of axon branching in a systematic manner, as, for example, sprouting and growth of a significant number of axon branches in a defined direction: tortuosity (actual branch length per linear distance between branch endpoints), planar angle (change in direction that the branch makes with respect to the previous branch), and $X Y$ angle and $Z$ angle (together these two angles are the spherical coordinate angles for the end of the branch relative to the start of the next branch).

Analysis was concentrated on the three lower centripetal branch orders. The centripetal branch order is defined as the shortest distance of a branch from an ending, in terms of number of intervening connecting nodes. In this way, terminal branches have centripetal order $0(\mathrm{c} 0)$, branches connected to $\mathrm{c} 0$ have order $\mathrm{cl}$, and so on. We examined the differences of branches of order $\mathrm{c} 0-\mathrm{c} 2$ between axons terminating in the normal and deafferented parts of VP lateral nucleus (VPL) or SI using multivariate logistic regression analysis in SPSS 15.0. This analysis allowed us to control for increased risk of type I error deriving from multiple univariate tests. The goodness-offit of the model was assessed with the Hosmer and Lemeshow test. The variance in the observed data explained by the logistic regression model was estimated by the Nagelkerke's $R^{2}$ test. We used partial correlation analysis to assess variable interactions. The measured variables were entered in the logistic regression model as predictors and the locus of axon termination (deafferented versus normal) as the binary outcome (see Fig. 3). The predictors were entered in the model in two blocks. Block 1 included the first group of variables described above. Block 2 included the second group of variables, which we did not expect to be significantly impacted by the atrophic processes, but 

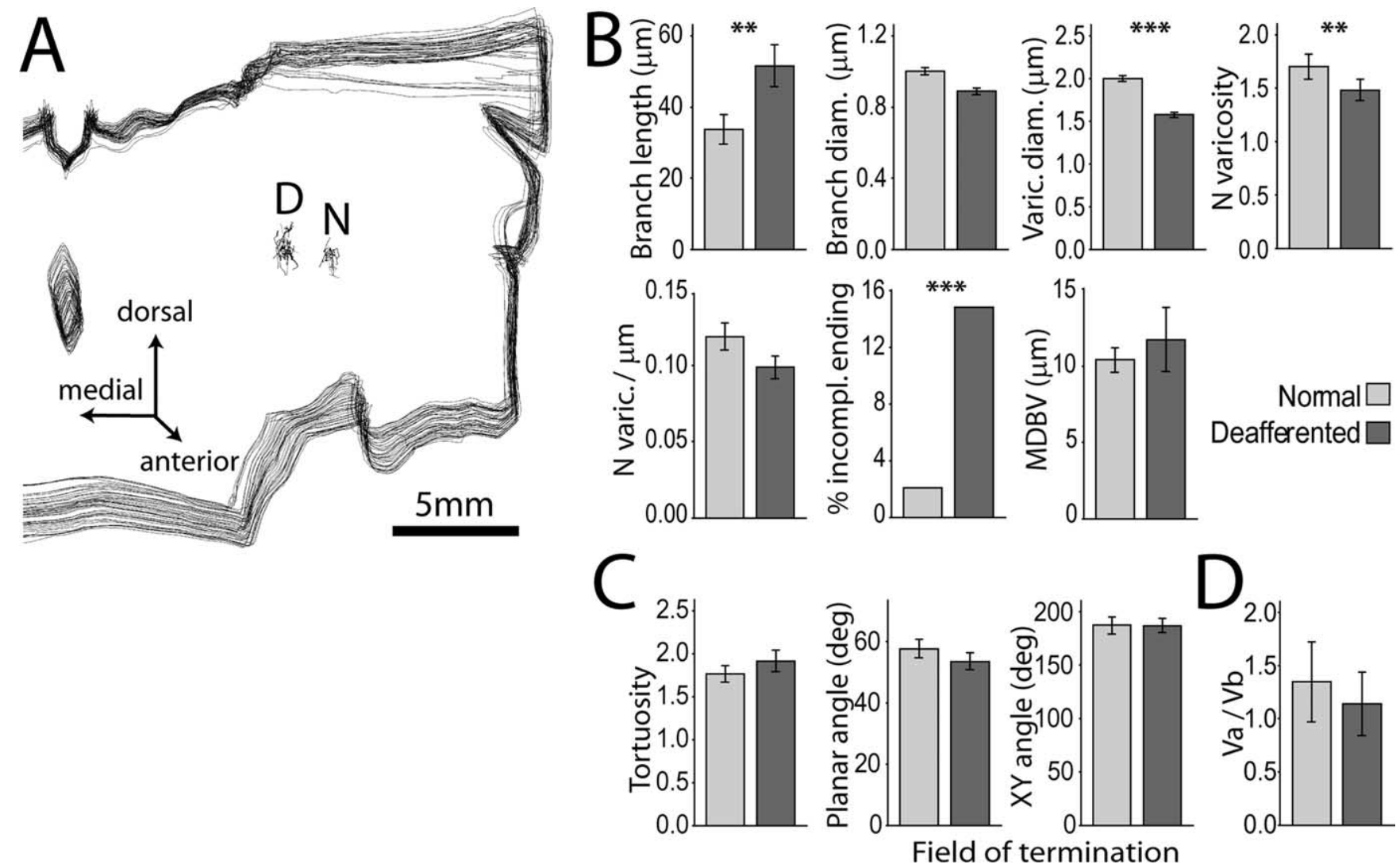

Figure 3. Somatosensory deafferentation causes reduction in the size and number of lemniscal synaptic boutons in VPL. $A$, Three-dimensional reconstruction of a stack of 45 consecutive coronal sections through the posterior thalamus of case RM141, showing the location of two clusters of axons terminating in separate fields of VPL: D, deafferented field; $N$, normal field. Differences between terminal branches and associated synaptic boutons in the two groups of axons were examined by logistic regression. $\boldsymbol{B}$, Block 1 variables. The largest effects were reduction in varicosity size ( $-20 \%$; Wald $=91.259, p=0.000 ; \operatorname{Exp}(B)=0.007)$ and number $(-11.8 \% ;$ Wald $=8.651, p=0.003 ; \operatorname{Exp}(B)=0.707)$ in the deafferented region. Deafferentation also caused a significant increase in the number of incomplete endings (Wald $=6.733, p=0.009 ; \operatorname{Exp}(B)=4.818)$ and smaller increase in branch length (Wald $=7.694, p=0.006 ; \operatorname{Exp}(B)=1.015)$. C, None of the variables in Block 2 displayed significant differences between the two groups of axons. $D$, Vertex analysis showed no difference in the $V_{A} / V_{B}$ vertex ratio (ANOVA $F_{(1,59)}=2.974$, nonsignficant). Mean $\pm 2 \times$ SEM. ${ }^{* *} p<0.01 ; * * * 0.001$.

in which we would possibly detect significant growth of axon branches with a directional bias.

Vertex analysis (Sadler and Berry, 1983; Berry and Flinn, 1984; Berry et al., 1986) was performed on the axon terminal arborizations in Neuroexplorer. This analysis provided information about the topology of branching structures. It calculates the ratio between nodes that have two terminating branches attached, $\mathrm{V}_{\mathrm{A}}$, and the nodes that have one terminating branch attached, $\mathrm{V}_{\mathrm{B}}$. Trifurcating nodes are transformed in bifurcating nodes. A value $>1$ suggests that the tree is nonrandom and symmetrical. Values $\sim 1.0$ suggest that terminal nodes grew in random processes. Values $\sim 0.5$ suggest that segment growth grew the tree. A value $<0.5$ suggests the tree is nonrandom and asymmetrical. Parts of this description were extrapolated from the Neuroexplorer tutorial on vertex analysis (MBF Bioscience). The means of $\mathrm{V}_{\mathrm{A}} / \mathrm{V}_{\mathrm{B}}$ ratios of axons terminating in normal or deafferented regions of thalamus and cortex were tested by univariate ANOVA.

Images. Images of axon terminals were acquired with the same system used for axon tracing (see above). Images of axon tracings were exported as bitmap files from Neuroexplorer. Images of immunostained spinal cord section were obtained by a Quantix CCD camera (PhotoMetrics) interfaced to a personal computer and operated by SimplePCI software (Compix) attached to a Nikon Eclipse 1000 microscope. Image files were imported into Photoshop CS2 (Adobe Systems), cropped, and contrast adjusted. Graphs were generated in SPSS, saved as Windows metafiles (WMF), and imported into Adobe Illustrator. The adjusted pictures, graphs, and drawings were composed in Illustrator and finally edited in Photoshop for publication.

\section{Results}

In all 10 monkeys, two years after surgical division of the left cuneate fasciculus at the $\mathrm{C} 1$ level of the spinal cord (Fig. 1A), the degeneration of the central portions of the severed axons had led to almost complete loss of the cuneate fasciculus above the level of the lesion (Fig. $1 B$ ). Two years after a spinal injury of this type, no clear cellular atrophy has been reported in the brainstem or thalamus (Cowan et al., 1970), and that was confirmed in the present study, but cortical reorganization is demonstrable (Merzenich et al., 1984; Jain et al., 2008). At this point, we injected the anterograde tracer, $\mathrm{BDA}$, in the dorsal column nuclei (intact gracile and denervated cuneate nuclei) (Fig. 1A) of one or both sides, or in the VP nucleus of the thalamus, to study the morphology of labeled lemniscal axon terminals in the thalamus (Figs. 2, 3; also see Fig. 6) or of thalamocortical axons in area $3 \mathrm{~b}$ of the somatosensory cortex (see Fig. 7). The total length of reconstructed axon terminations (Fig. 1) was $435,623.3 \mu \mathrm{m}$, corresponding to $>25,000 \mathrm{~m}$ (15.5 miles) of tracings at the working magnification $(100 \times)$; they bore 16,801 varicosities, representing both en-passant and terminal synaptic boutons (Westrum and Blackstad, 1962; Pichitpornchai et al., 1994).

The stereotypical topography of body representations along the lemniscal somatosensory pathway (Jones, 2007) al- 
Table 1. Partial correlation analysis showing that the difference in branch diameter is caused entirely by the difference in varicosity diameter

\begin{tabular}{|c|c|c|c|c|c|}
\hline \multicolumn{6}{|l|}{ A } \\
\hline \begin{tabular}{|l} 
Control \\
Variable
\end{tabular} & & & $\begin{array}{l}\text { Predicted } \\
\text { probability }\end{array}$ & $\begin{array}{l}\text { Varicosity } \\
\text { diameter }\end{array}$ & $\begin{array}{c}\text { Branch } \\
\text { diameter }\end{array}$ \\
\hline \multirow[t]{9}{*}{-none-(a) } & \multirow{3}{*}{\begin{tabular}{|l|} 
Predicted \\
probability
\end{tabular}} & Correlation & 1.000 & -0.896 & -0.634 \\
\hline & & Significance (2-tailed) & & 0.000 & 0.000 \\
\hline & & df & 0 & 624 & 624 \\
\hline & \multirow{3}{*}{$\begin{array}{l}\text { Varicosity } \\
\text { diameter }\end{array}$} & Correlation & -0.896 & 1.000 & 0.700 \\
\hline & & Significance (2-tailed) & 0.000 & & 0.000 \\
\hline & & df & 624 & & 624 \\
\hline & \multirow{3}{*}{$\begin{array}{l}\text { Branch } \\
\text { diameter }\end{array}$} & Correlation & -0.634 & 0.700 & 1.000 \\
\hline & & Significance (2-tailed) & 0.000 & 0.000 & \\
\hline & & df & 624 & 624 & \\
\hline
\end{tabular}

\section{B}

\begin{tabular}{|l|l|l|r|r|r|}
\hline \multirow{3}{*}{$\begin{array}{l}\text { Branch } \\
\text { diameter }\end{array}$} & $\begin{array}{l}\text { Predicted } \\
\text { probability }\end{array}$ & Correlation & 1.000 & -0.818 & \\
\cline { 3 - 6 } & & Significance (2-tailed) & & 0.000 & \\
\cline { 2 - 6 } & df & -0.818 & 1.000 & \\
\cline { 2 - 6 } & $\begin{array}{l}\text { Varicosity } \\
\text { diameter }\end{array}$ & Correlation & 0.000 & & \\
\cline { 3 - 6 } & & Significance (2-tailed) & 623 & & \\
\cline { 3 - 6 } & & df & & & \\
\hline
\end{tabular}

\section{C}

\begin{tabular}{|c|c|c|c|c|}
\hline \multirow{6}{*}{$\begin{array}{l}\text { Varicosity } \\
\text { diameter }\end{array}$} & \multirow{3}{*}{$\begin{array}{l}\text { Predicted } \\
\text { probability }\end{array}$} & Correlation & 1.000 & -0.023 \\
\hline & & Significance (2-tailed) & & 0.561 \\
\hline & & df & 0 & 623 \\
\hline & \multirow{3}{*}{$\begin{array}{l}\text { Branch } \\
\text { diameter }\end{array}$} & Correlation & -0.023 & 1.000 \\
\hline & & Significance (2-tailed) & 0.561 & \\
\hline & & df & 623 & \\
\hline
\end{tabular}

A, Zero-order correlations: both branch diameter and varicosity diameter correlate significantly with the predicted probability of the logistic regression model and with each other (highlighted in red). a, Cells contain zero-order (Pearson) correlations. B, After controlling for branch diameter, partial correlation between varicosity diameter and predicted probability is still highly significant, with only a marginal decrease of the correlation coefficient. C, After controlling for varicosity diameter, partial correlation between branch diameter and predicted probability is not significant (highlighted in blue), with a correlation coefficient close to zero.

lowed us to compare the morphology of axons terminating in the deafferented regions of the VPL or of area $3 \mathrm{~b}$, with those terminating in unaffected regions (Figs. $1 A, 2,3 A$ ). The boundary between deafferented and normal representations in VPL or in area 3b was identified electrophysiologically before terminating the experiment (see Figs. 5, 6).

\section{Electrophysiological mapping of deafferented DCN, VP, and SI}

The cuneate nucleus was virtually silenced by the denervation, whereas the adjacent gracile nucleus displayed normal lowthreshold neuronal responses to peripheral stimulation. The priority of the study of preserving the physical integrity of cells and axons in the dorsal column nuclei (DCN) to trace their terminations in VPL precluded a fine-grained mapping of receptive fields in the brainstem. Nonetheless, we systematically stimulated the face during all electrode penetrations in the DCN and never found face receptive fields (RFs) in the silenced cuneate nucleus or more medially in the gracile nucleus. Neuronal responses evoked by stimulation of the face were only recorded in more lateral and deeper penetrations, which reached the caudal portion of the spinal trigeminal nucleus.

The response properties of cells in the VPL of the thalamus and in SI mirrored those of cells in the cuneate and gracile nuclei. In VPL, the region receiving fibers from the deafferented cuneate nucleus was virtually unresponsive to light touch or hair displacement and was located between two regions with normal, lowthreshold neuronal responses and small RFs: a medial region containing neurons with receptive fields on the face, head, and neck (VPM), and a lateral region, containing neurons with receptive fields on the leg, tail, and lower trunk (the lateral portion of VPL, receiving input from the intact gracile nucleus) (see Figs.

$1 A, 6 A, B)$. We did not observe frank expansion of the normal trigeminal or gracile representations into the deafferented hand region of VPL; face RFs were never in close proximity to leg RFs, as observed after much longer post-denervation times (Jones and Pons, 1998).

In the cortex, the hand/face border, as defined by mapping the face representation, was located at a level opposite the lateral tip of the intraparietal sulcus (see Fig. $7 A, B$ ), as normally found in adult macaque monkeys (Dreyer et al., 1975; Pons et al., 1991). Medial to this, in the deafferented hand area, electrophysiological activity after peripheral stimulation was remarkably similar to that in the deafferented thalamus, with a majority of unresponsive sites, some weak evoked activity with very large receptive fields, at times including the whole hand and forearm, and responses elicited only by heavy tapping. To avoid compromising the labeling and reconstruction of thalamocortical axons, we did not carry out extensive microelectrode penetrations of the cortex, so we did not acquire data about the extent of enlargement of the face representation

\section{Axon morphology quantification}

Visual inspection of terminal arbors of axons labeled by anterograde transport from the denervated cuneate neurons ending in the upper limb representation of the VPL, or of terminal arbors of axons of denervated VPL neurons ending in area $3 \mathrm{~b}$ suggested shrinkage in comparison with the terminal arbors of axons arising from neurons in the non-deafferented gracile nucleus or intact lower limb representation of VPL (Fig. 2). The appearance resembled very closely that of geniculocortical axons shrinking in the visual cortex in response to visual deprivation during the critical period (Antonini and Stryker, 1993). In deafferented regions of thalamus and cortex, a higher number of labeled axonal processes terminated abruptly, lacking terminal synaptic boutons typically observed in normal axon terminals. These incomplete endings did not display morphological characteristics suggestive of growth cones, nor did they possess the dystrophic morphology observed when regenerating axons in the CNS come to a halt after reaching the glial scar in vivo or in vitro (Bandtlow et al., 1990; Burden-Gulley et al., 1995; Li and Raisman, 1995).

The vertex analysis performed on the two groups of reconstructed axons has previously been used to describe morphological plasticity of dendritic trees during development (Sadler and Berry, 1983; Berry and Flinn, 1984; Berry et al., 1986). Vertex analysis, which does not take into account metric properties of branching structures, did not detect the differences in gross topology observed qualitatively (Fig. 2) between axons in normal and deafferented regions of thalamus (Fig. 3D) or cortex (Fig. 7E). However, the terminal morphology of the axons showed highly significant changes when examined using multivariate logistic regression analysis, with the measured variables entered in the model as predictors and the locus of axon termination (deafferented vs normal) as the binary outcome (Fig. 3). The predictors were entered in the model in two blocks. Block 1 included predictors relevant to the hypothesis of axon withdrawal from VPL resulting from transneuronal atrophy of parent cells in the cuneate nucleus: branch length and diameter, number and density of varicosi- 


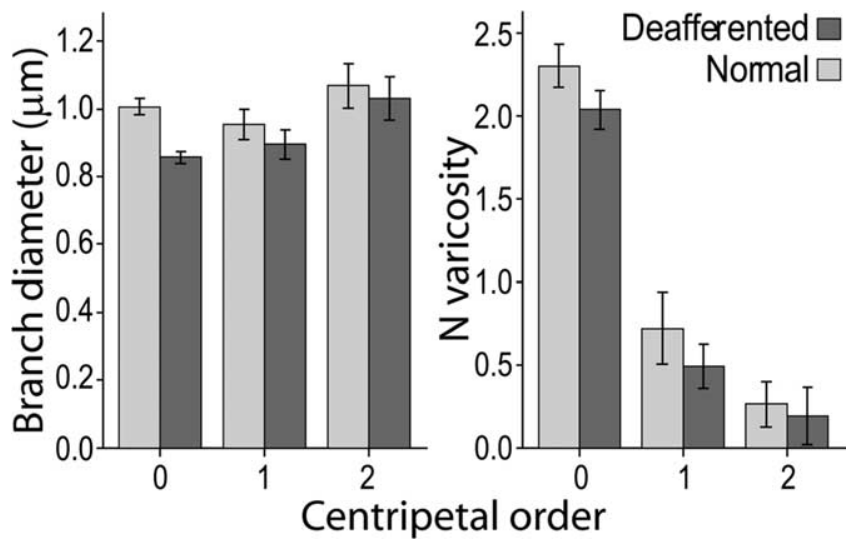

Figure 4. Branch diameter and number of varicosities in branches of different centripetal orders. The difference in branch diameter between axons terminating in normal and deafferented VPL is maximal for the terminal branches (top), reflecting the number of varicosities per branch, which is progressively smaller with the increase of the topological distance (centripetal order) from the axon ending (centripetal order $=0$ ) to more proximal branches. Mean $\pm 2 \times$ SEM.

ties per branch, varicosity diameter, median distance between varicosities, and number of incomplete endings. Block 2 included variables that we did not expect to be significantly impacted by an atrophic process, such as tortuosity, planar angle, $X Y$ angle, and $Z$ angle.

Only the Block 1 contribution to the model was highly significant (omnibus test of model coefficients, $X^{2}=283.682, p=$ $0.000)$. Adding Block 2 to the analysis did not improve the model by any significant measure $\left(X^{2}=1.426, p=0.840\right)$. The Hosmer and Lemeshow test was not significant $\left(X^{2}=7.479, p=0.486\right)$, indicating the good fit of the model to the observed data. The estimated variance explained by the model was almost $50 \%$ (Nagelkerke $R^{2}=0.478$ ), leading to correct classification of $72.6 \%$ of cases in the normal part of VPL and $80.0 \%$ in the deafferented part (average: $76.5 \%$ ).

Analysis of the single variables in the model (Fig. $3 B, C$ ) showed the largest effect was a $20 \%$ reduction in boutonal size in the deafferented part of VPL compared with the nondeafferented part. Although the average branch diameter was $10 \%$ smaller in the deafferented part, this difference was not significant, likely a consequence of the variance shared with varicosity size, as shown by the partial correlation analysis of these two variables with the predicted probability of the model (Tables 1). This conclusion is supported by the fact that the difference was maximal in the terminal branches (centripetal order $=0$ ), which bear the largest number of synaptic boutons (Fig. 4). A significant increase in branch length in the deafferented part of VPL can be explained by the reduction in number of the shortest branches in the distal part of the axon (Fig. 5). The primary involvement of synaptic terminals in the atrophic process was confirmed by the significant reduction of bouton number $(-11.8 \%)$ and higher number of incomplete endings $(12.7 \%)$.

The lack of significant improvement after entering Block 2 in the model $\left(X^{2}=1.426, p=0.840\right)$ is in line with the outcome of vertex analysis, indicating that the gross geometry of terminal branching is relatively unaffected up to two years after deafferentation. The first detectable effects of ascending transneuronal atrophy instead consist of a substantial reduction in size and number of lemniscal synapses in the deafferented thalamus.

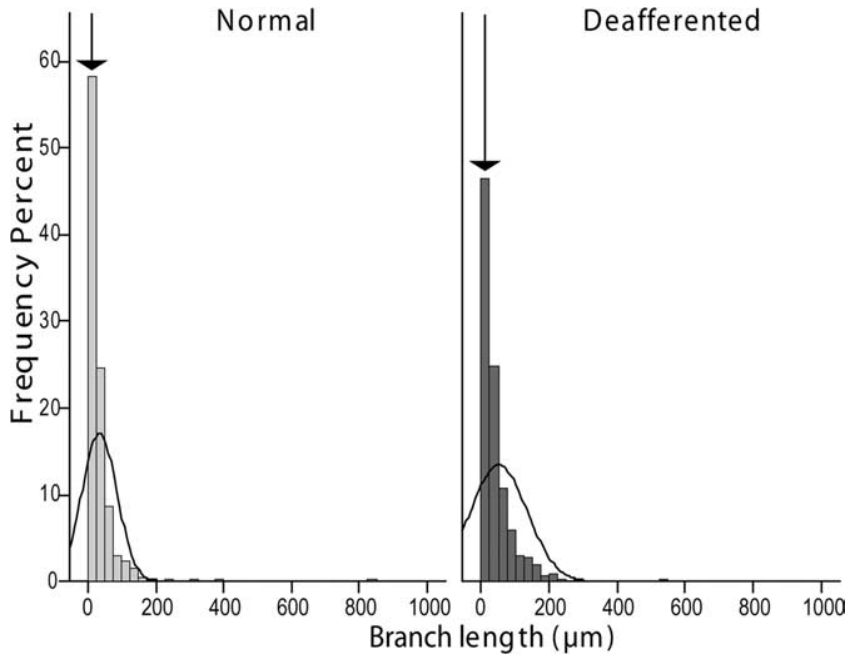

Figure 5. Lemniscal axon withdrawal affects mostly the terminal branches. Histograms showing the relative distribution of axon branch lengths in the normal and deafferented fields of termination in VPL. The arrows point to the bar corresponding to the shortest branches, which are less numerous in the deafferented VPL, suggesting that many small-sized branches are lost in this area.

This result was reflected in the electrophysiological mapping of somatosensory evoked multiunit responses in the deafferented part of VPL, described in the previous section on electrophysiological mapping (Fig. 6).

Clusters of anterogradely labeled axon terminals from electrophysiologically characterized regions of VPL were reconstructed (Fig. 6). In the deafferented part of VPL, the average size of synaptic boutons was reduced by $31.8 \%$ relative to those in the unaffected part (Fig. $6 C-E$ ), a larger difference compared with that observed after sampling axons solely on the base of topographic criteria (Fig. 3). Logistic regression model in this case explained $>85 \%$ of the variance (Nagelkerke $R^{2}=0.861$ ) and led to $93.7 \%$ correct classification. As in the previous analysis, model performance was due entirely to Block $1\left(X^{2}=199.572, p=0.000\right)$; the contribution of Block 2 variables was not significant $\left(X^{2}=4.531\right.$, $p=0.339)$ and did not produce any increment of the model's predictive power obtained with Block 1 alone (93.7\% correct case classification).

The same approach was used to investigate morphological changes of thalamocortical axons ending in the deafferented hand representation of area $3 \mathrm{~b}$ with those ending in the face representation, unaffected by the cuneate lesion (Fig. 7). Anterogradely labeled axons taken from either side of the electrophysiologically mapped hand/face border were compared. The results of logistic regression analysis of labeled axon terminations (Fig. 6C,D) were virtually identical to those obtained in the thalamus, with the largest effects consisting in reduction of boutonal size $(-21.7 \%)$ and number $(-40 \%)$, and increase in the number of incomplete endings on terminations in the deafferented hand area (1.3\% normal vs $8.4 \%$ deafferented) in comparison with axons from the face area.

\section{Discussion}

The results demonstrate that after massive peripheral denervation, transneuronal atrophy affects the morphology of second order lemniscal and third order thalamocortical axons long before overt shrinkage of their deafferented parent cells in dorsal column nuclei or thalamus. This shrinkage, with 

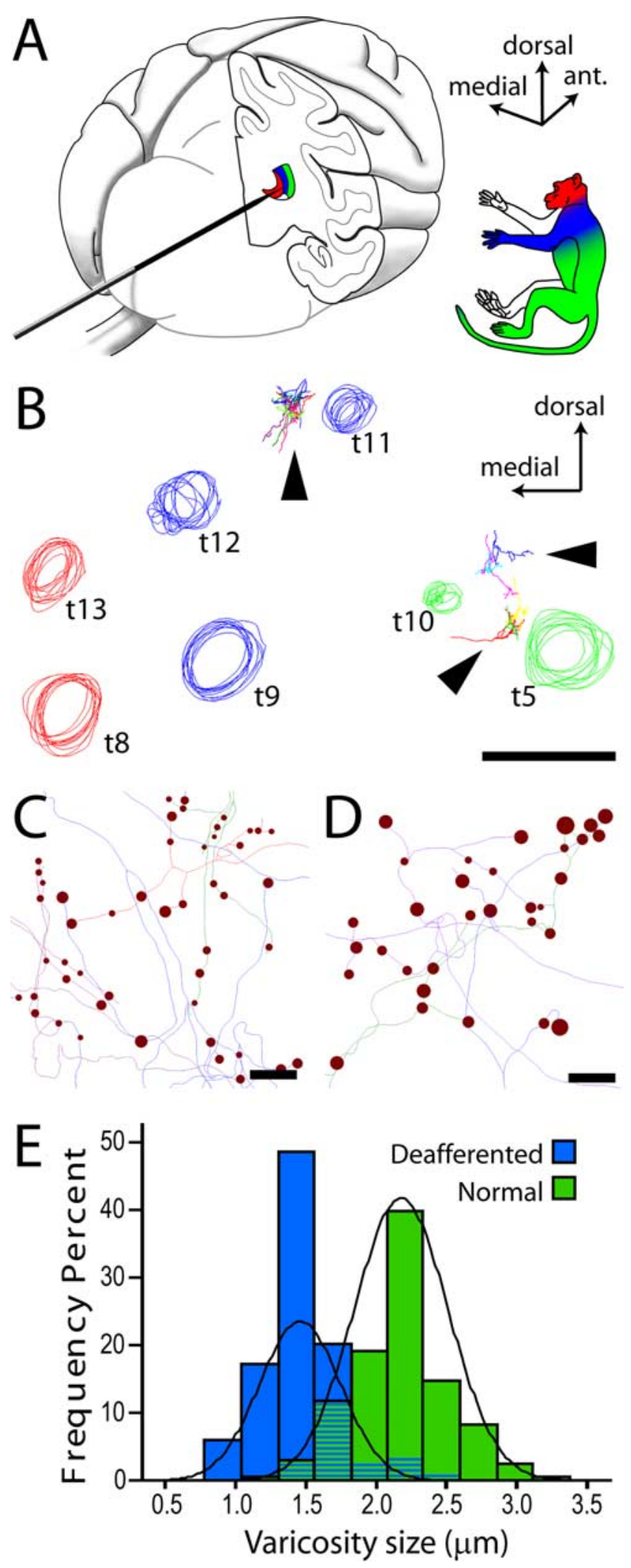

Figure 6. Axon tracing from electrophysiologically characterized regions of VP. A, The electrode was inserted into the brain from the occipital pole and advanced after a horizontal trajectory. Multiunit evoked responses from multiple electrode penetrations were used to identify the somatotopic map in VP. B, Circles represent tracks left by an array of electrode penetrations, reconstructed from a series of coronal sections through the posterior thalamus. Track colors represent the portion of periphery from which evoked activity could be recorded. The majority of sites in the hand representation (blue) were silenced by the spinal lesion. Arrowheads point to labeled axons. Axons reconstructed from the deafferented hand field (between blue tracks t11 some loss of neurons, can be detected only after deafferentation periods of 10 or more years (Woods et al., 2000). The effects of the shorter term deafferentation on the terminal arbors of axons in thalamus and cortex are similar, consisting primarily of a considerable reduction in size and number of synaptic boutons.

\section{Axon withdrawal can explain cortical reorganization}

As a consequence of the extensive divergence of ascending axons at all levels of the somatosensory system (Jones et al., 1997; Rausell et al., 1998), even small changes in the innervation of the dorsal column nuclei will be magnified at successively higher levels up to the cortex. In the monkey thalamus, $0.1 \mathrm{~mm}^{3}$ of the ventral posterior nucleus projects to as much as $20 \mathrm{~mm}^{2}$ of cortical surface (Rausell et al., 1998), so that subthreshold input from a single digit can normally extend over many millimeters of SI, a much wider area than that detected by extracellular mapping. Because of this wide divergence, a large proportion of the thalamic representation of the hand can be destroyed before the representation of a digit, as mapped by extracellular recording, begins to shrink (Jones et al., 1997). Loss of input activity to one region of the cortical somatosensory map by withdrawal of synaptic terminations from axons affected by transneuronal atrophy of the kind described will, therefore, unmask existing latent synapses (Wall, 1977) that carry information relative to adjacent body parts (Schroeder et al., 1995), and will reveal itself in expansion of the unaffected representation (Fig. 8).

Over the long term, continuing secondary transneuronal atrophy of neurons in the thalamus after primary transneuronal atrophy of neurons in the cuneate nucleus causes the deafferented part of VPL physically to shrink, bringing cells with receptive fields on the face, normally contained within the VPM nucleus, in close proximity to cells with trunk and lower limb receptive fields (Jones and Pons, 1998; Woods et al., 2000). Similar changes occur in the human thalamus after long-term deafferentation (Lenz et al., 1994; Draganski et al., 2006). When coupled with the divergence of intact inputs from below, this shrinkage can lead to large shifts of the preserved representations into adjacent deafferented fields, beyond the cortical distance limit (Jones et al., 2002), even in the absence of newly formed long-range sprouting from intact axons in the brainstem or cortex. Sprouting has hitherto been represented as a leading mechanism for inducing cortical reorganization (Buonomano and Merzenich, 1998; Merzenich, 1998; Darian-Smith and Brown, 2000; Kaas and Florence, 2001; Kaas et al., 2007).

\section{Is sprouting essential for cortical reorganization?}

Divergence of ascending input and unmasking of existing synapses may explain observations in a patient who reported precise, topographically organized phantom sensations after stimulation of the face as early as $24 \mathrm{~h}$ after arm amputation

and $\mathrm{t} 12$ ) were compared with axons terminating in the leg/lower body area (between green tracks $t 5$ and t10). $\mathbf{C}, \mathbf{D}$, Line rendering of lemniscal axon tracings from deafferented $(\boldsymbol{C})$ and normal (D) VPL, showing the intrinsic size of synaptic boutons (varicosities). Scale bar in $\boldsymbol{D}$ applies also to $\boldsymbol{C}$. $\boldsymbol{E}$, Frequency distributions of varicosity sizes in the two groups of axons. The average reduction of varicosity size in deafferented VPL was $31.8 \%$ (Wald $=22.740, p<$ $0.001, \operatorname{Exp}(B)=0.000)$. 


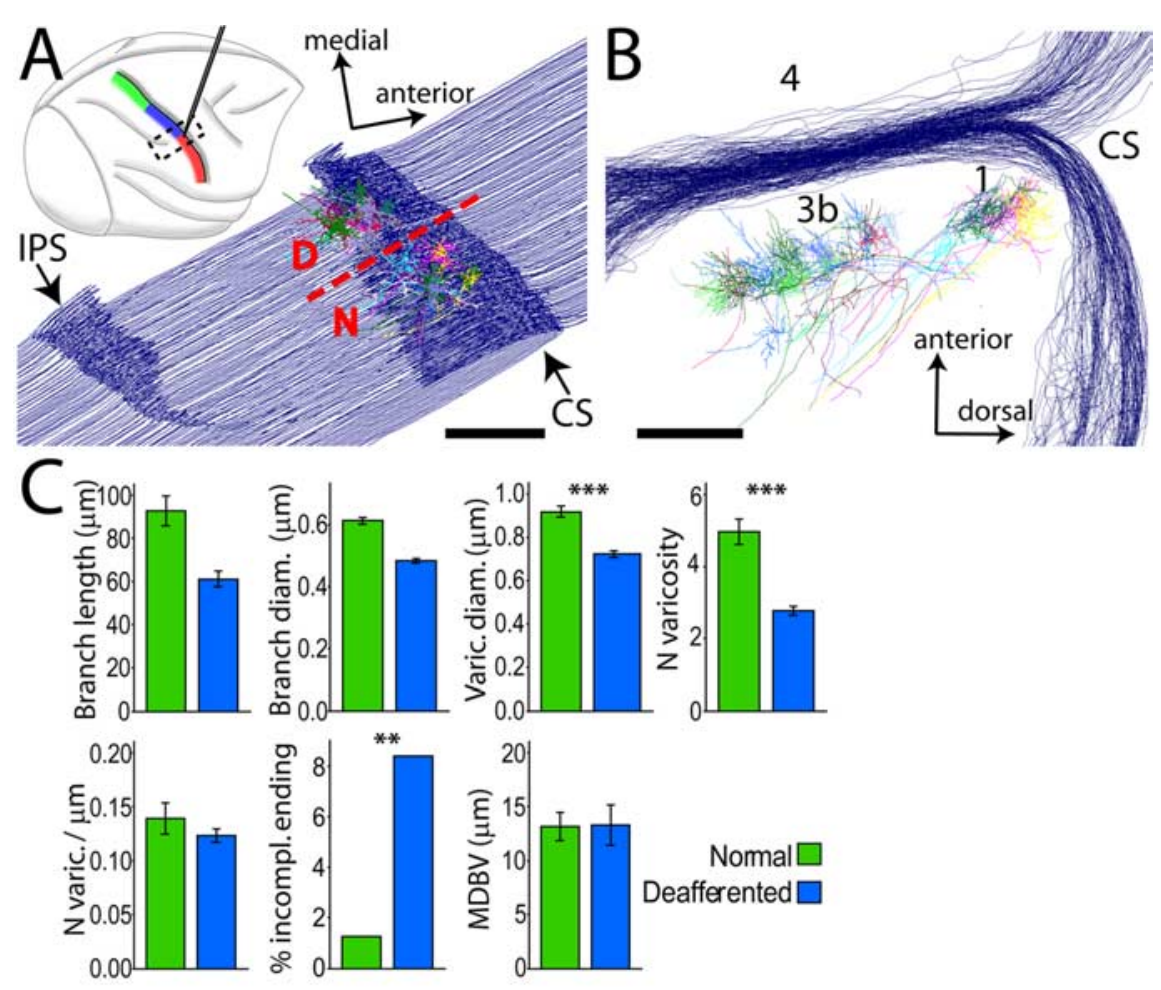

$\mathrm{D}$
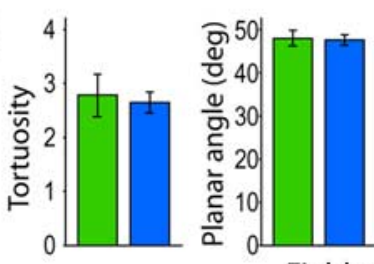

Field of termination

Figure 7. Somatosensory deafferentation causes reduction in the size and number of thalamocortical synaptic boutons in SI. $\boldsymbol{A}$, Top, Right hemisphere, lateral view. Electrophysiological mapping of somatosensory representations in the postcentral gyrus, color coded as in Figure 1. Electrode tracks were used to identify the border (dashed line in bottom diagram, at the level of the lateral tip of IPS) between normal (N) face and deafferented (D) hand representations, from which two groups of thalamocortical axons were reconstructed. Dashed box in top diagram shows location and orientation of the series of cortical sections used for axon reconstruction. $\boldsymbol{B}$, Same reconstruction as in $\boldsymbol{A}$, rotated $90^{\circ}$ around its anteroposterior axis to show the blockface view, illustrating axons terminating in layer IV of areas $3 \mathrm{~b}$ and 1 . C, Block 1 variables of logistic regression model. As in VP, the largest effect was a significant reduction in size $(-21.7 \%$; Wald $=$ $314.186, p=0.000 ; \operatorname{Exp}(B)=0.000)$ and number $(-40 \% ;$ Wald $=33.226, p=0.000 ; \operatorname{Exp}(B)=0.903)$ of varicosities in deafferented $\mathrm{SI}$, and a significant increase in the number of incomplete endings. $\boldsymbol{D}$, No significant difference was observed among Block 2 variables. $\boldsymbol{E}$, Vertex ratio did not differ in the two fields of termination. Mean $\pm 2 \times$ SEM. ${ }^{* *} p<$ $0.01 ;{ }^{* * *} p<0.001$.

(Borsook et al., 1998) and the demonstration of cortical reorganization $1 \mathrm{~h}$ after pharmacological block of the median nerve (Weiss et al., 2004). Moreover, in amputees suffering from phantom pain, peripherally or centrally induced analgesia can cause immediate and temporary normalization of cortical maps, with concomitant release from pain in the phantom limb (Birbaumer et al., 1997; Huse et al., 2001). These and other clinical observations (Ramachandran et al., 1992; Knecht et al., 1998), although not dismissing the possibility of sprouting at all levels of the somatosensory pathways after deafferentation, question a causal role for newly formed longrange projections in cortical reorganization (Knecht et al., 1998). Recent experimental data reinforce this view. Two years after lesioning the cuneate fasciculus at $\mathrm{C} 5-\mathrm{C} 7$ in adult monkeys, Jain at al. (2008) observed massive reorganization in SI, finding neurons with face receptive fields as far medially as the representation of the foot. The foot area receives input from the gracile nucleus via VPL. It is significant that, at comparable postlesion times, sprouting of spinal trigeminal axons after more massive deafferentations, produced by lesioning both cuneate and gracile fasciculi at higher cervical levels (C3-C5), was limited to the cuneate nucleus (Jain et al., 2000), making it unlikely that cortical reorganization depends solely on axonal sprouting in the brainstem. The evidence that newly formed dorsal column axons reaching the cuneate nucleus after spinal cord lesion are chronically demyelinated and remain in a pathophysiological state (Tan et al., 2007) demands careful consideration of the functional significance of sprouting in cortical reorganization. It is also apparent, from the absence of morphological features typical of growing axons in our experiments, that sprouting, successful or failed, of lemniscal or thalamocortical axons does not represent a significant, if at all detectable, mechanism of cortical reorganization at this time. In the cortex itself, the sprouting demonstrated after massive deafferentation (Florence et al., 1998) is not sufficiently extensive to explain the expansion of the face representation in SI described by Jain et al. (2008).

\section{Early reorganization may depend on preserved cuneate input}

Two years after deafferentation there was no noticeable replacement of the deafferented limb representation by the face representation in the thalamus. In the deafferented region, neurons were unresponsive to light touch or hair displacement and responses could only be evoked by heavy tapping, likely mediated by preserved spinothalamic inputs. We did not extensively map the cortical representation to preserve the integrity of the labeled axons, but medial to the face representation in the deafferented upper limb representation, neurons were as little responsive as in the thalamus. Other studies reported massive expansion of the cortical face representation after lesion of the dorsal columns at levels varying from C3 to C7 (Jain et al., 1997; Jain et al., 2008). In primates, the transverse cutaneous nerve of the neck, whose axons enter the spinal cord at $\mathrm{C} 2-\mathrm{C} 3$ and terminate in the cuneate nucleus, extends its innervation field from the neck into the face as far as the chin, overlapping the field innervated by the mandibular branch of the trigeminal nerve (Sherrington and Denny-Brown, 1939; Carpenter and Sutin, 1983). This implies that lesions at or below the $\mathrm{C} 3$ level will preserve input from this part of the face in the cuneate nucleus. Because we interrupted the cuneate fasciculus at $\mathrm{Cl}$, we effectively removed all input to the $\mathrm{cu}$ - 
neate nucleus, the preparation more closely resembling the massive denervation performed by cutting the dorsal roots from C2 to T4 (Pons et al., 1991). After complete lesions of this kind (Bioulac and Lamarre, 1979), the area of cortex receiving cuneate input is completely silenced, with the exception of a small region receiving head input behind the arm representation, present in normal monkeys (Ullrich and Woosley, 1954; Dreyer et al., 1975). Unfortunately, the only data on cortical reorganization available from 6 to 8 months to 2 years and thus relevant to the present study were obtained after lesions at levels ranging from $\mathrm{C} 3$ to $\mathrm{C} 7$, so they do not shed light on this issue. It must be noted that even after more restricted digit deafferentations, areas of silenced cortex persist for as long as 32 weeks, and the extent of cortical reactivation depends on the size of the deafferenting lesion (DarianSmith and Ciferri, 2006). The present observations suggest that after complete cuneate deafferentation, thalamic and cortical hand representations may remain silent for years before becoming occupied by inputs from the face.

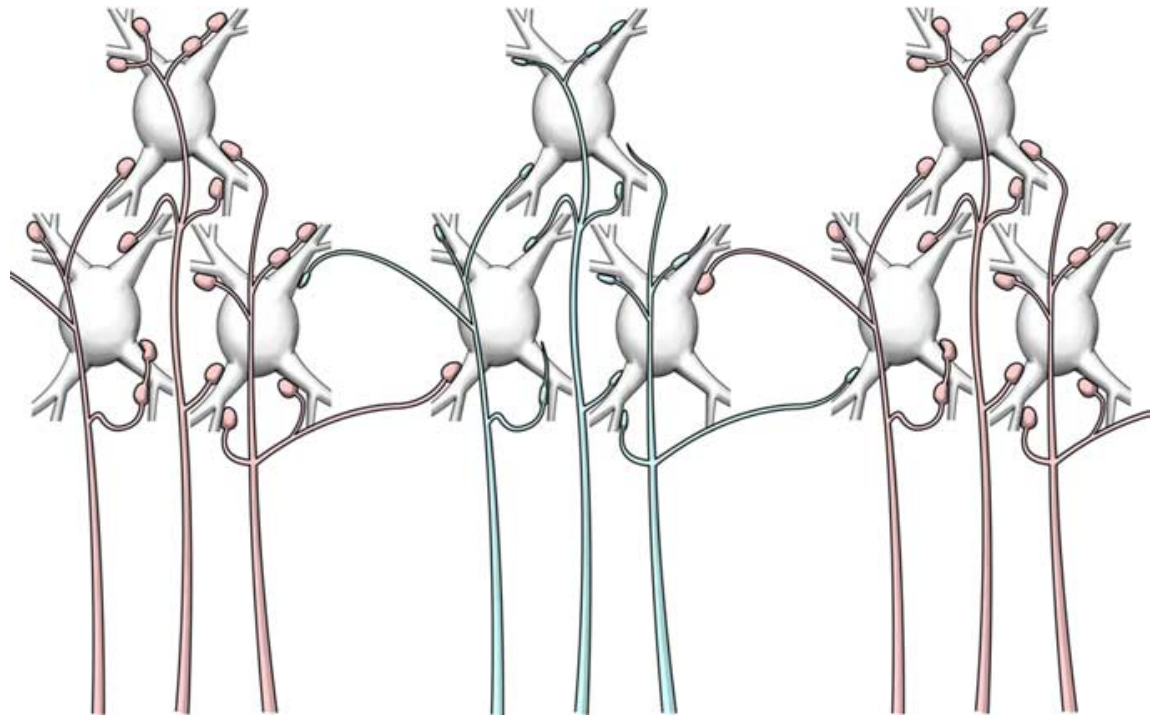

Figure 8. Diagram summarizing the morphological changes of withdrawing lemniscal axons (blue) from the upper limb and upper trunk representations in VPL after transection of the cuneate fasciculus. The same scheme applies to thalamocortical axons withdrawing from the upper limb and upper trunk representation in the somatosensory cortex. The first signs of transneuronal atrophy are a reduction in size and number of synaptic boutons, the presence of incomplete endings, and a loss of short terminal branches. The divergent projections of normal axons (red) from face and lower body representations to the adjacent cuneate representations, normally unable to drive activity, constitute the basis for the expansion of the silenced upper limb/upper trunk representation by adjacent representations of the face and lower body. In the long term, transneuronal atrophy, with shrinkage and loss of deafferented neurons, enhances the expansion of representations with intact innervation.

\section{Axon withdrawal is progressive}

A reported lack of transneuronal atrophy in earlier studies of peripheral deafferentation is the consequence of focusing only on somal morphology. The lack of somatic shrinkage in the short term has led to the dismissal of transneuronal atrophy as a contributor to cortical reorganization (Kaas and Florence, 2001). In shifting the focus from cell body to axon, the present results demonstrate that even at early postlesional stages, transneuronal atrophy has affected lemniscal and thalamocortical axon terminations, suggesting that transneuronal atrophy and withdrawal of the axons of deafferented neurons is likely to contribute to cortical reorganization even in the short term. The presence of axon withdrawal from thalamus and cortex early after deafferentation, together with the evidence of lemniscal axon loss in VPL (Jones and Pons, 1998) and cortical atrophy in the somatosensory cortex (Campbell, 1905) after very long-term deafferentation, indicate the progressive nature of the ascending transneuronal effect and help explain the expansion of the face representation into the deafferented representation years after massive deafferentation.

\section{Peripheral denervation may induce similar changes}

The present study focused on the effects of deafferentation caused by cutting the central axons of dorsal root ganglion cells. Transneuronal atrophy of axons before it becomes manifest in somal shrinkage may also affect dorsal root ganglion cells after limb amputations or transections of nerves which sever the peripheral axons: in the long term, peripheral deafferentation causes extensive loss of dorsal root ganglion cells and of sensory axons entering the brainstem and spinal cord (Csillik et al., 1982; Knyihár-Csillik et al., 1987, 1989; Liss et al., 1996; Liss and Wiberg, 1997a,b), and transneuronal atrophy becomes detectable in neuronal somata in the cuneate nucleus and thalamus (Florence and Kaas, 1995; Jones and Pons, 1998; Woods et al., 2000) with a decrease in thalamic gray matter detectable by magnetic resonance imaging in humans (Draganski et al., 2006). The atrophy of cuneate cell bodies will be accompanied by changes of the type we have described in their axons terminating in the thalamus and across the next synapse in the axons of deafferented thalamic relay neurons in the cortex. Thus, peripheral and central deafferentation may act through the same mechanisms.

A neurodegenerative process, triggered by loss of somatosensory input that progresses over many years or decades is currently the only mechanism that can explain the long-term evolution of cortical reorganization and phantom sensations in spinal patients and amputees (Störmer et al., 1997; Hill, 1999). Deafferentation also leads to early, activity-dependent changes in expression of neurotransmitter, neurodegenerative, and neurotrophic genes in denervated regions (Graziano and Jones, 2006). The converging evidence of early denervation-induced regulation of genes related to neuronal atrophy and, at later stages, the progressive morphological changes of sensory axons and eventually cell atrophy and death in deafferented thalamus and cortex, suggest that longterm cortical reorganization and accompanying sensory phenomena, such as phantom limb and central pain, cannot be disassociated from a progressive neurodegenerative process that will be intertwined with and are likely to be a powerful inducement to adaptive plasticity.

\section{References}

Antonini A, Stryker MP (1993) Rapid remodeling of axonal arbors in the visual cortex. Science 260:1819-1821.

Bandtlow C, Zachleder T, Schwab ME (1990) Oligodendrocytes arrest neurite growth by contact inhibition. J Neurosci 10: 3837-3848. 
Berry M, Flinn R (1984) Vertex analysis of Purkinje cell dendritic trees in the cerebellum of the rat. Proc R Soc Lond B Biol Sci 221:321-348.

Berry M, Sadler M, Flinn R (1986) Vertex analysis of neural tree structures containing trichotomous nodes. J Neurosci Methods 18:167-177.

Bioulac B, Lamarre Y (1979) Activity of postcentral cortical neurons of the monkey during conditioned movements of a deafferented limb. Brain Res 172:427-437.

Birbaumer N, Lutzenberger W, Montoya P, Larbig W, Unertl K, Töpfner S, Grodd W, Taub E, Flor H (1997) Effects of regional anesthesia on phantom limb pain are mirrored in changes in cortical reorganization. J Neurosci 17:5503-5508.

Borsook D, Becerra L, Fishman S, Edwards A, Jennings CL, Stojanovic M, Papinicolas L, Ramachandran VS, Gonzalez RG, Breiter H (1998) Acute plasticity in the human somatosensory cortex following amputation. Neuroreport 9:1013-1017.

Buonomano DV, Merzenich MM (1998) Cortical plasticity: from synapses to maps. Annu Rev Neurosci 21:149-186.

Burden-Gulley SM, Payne HR, Lemmon V (1995) Growth cones are actively influenced by substrate-bound adhesion molecules. J Neurosci 15:4370-4381.

Calford MB (2002) Dynamic representational plasticity in sensory cortex. Neuroscience 111:709-738.

Campbell AW (1905) Histological studies on the localisation of cerebral function. Cambridge, UK: Cambridge UP.

Carpenter MB, Sutin J (1983) Human neuroanatomy. Baltimore: Williams and Wilkins.

Cowan WM, Nauta WJH, Ebbesson SOE (1970) Anterograde and retrograde transneuronal degeneration in the central and peripheral nervous system. In: Contemporary research methods in neuroanatomy, pp 217-249. New York: Springer.

Cowan WM, Südhof TC, Stevens CF (2003) Synapses. Baltimore: The Johns Hopkins University.

Csillik B, Knyihar E, Rakic P (1982) Transganglionic degenerative atrophy and regenerative proliferation in the Rolando substance of the primate spinal cord: discoupling and restoration of synaptic connectivity in the central nervous system after peripheral nerve lesions. Folia Morphol (Prague) 30:189-191.

Darian-Smith C, Brown S (2000) Functional changes at periphery and cortex following dorsal root lesions in adult monkeys. Nat Neurosci 3:476-481

Darian-Smith C, Ciferri M (2006) Cuneate nucleus reorganization following cervical dorsal rhizotomy in the macaque monkey: its role in the recovery of manual dexterity. J Comp Neurol 498:552-565.

Draganski B, Moser T, Lummel N, Gänssbauer S, Bogdahn U, Haas F, May A (2006) Decrease of thalamic gray matter following limb amputation. Neuroimage 31:951-957.

Dreyer DA, Loe PR, Metz CB, Whitsel BL (1975) Representation of head and face in postcentral gyrus of the macaque. J Neurophysiol 38:714-733.

Flor H, Elbert T, Knecht S, Wienbruch C, Pantev C, Birbaumer N, Larbig W, Taub E (1995) Phantom-limb pain as a perceptual correlate of cortical reorganization following arm amputation. Nature 375:482-484.

Florence SL, Kaas JH (1995) Large-scale reorganization at multiple levels of the somatosensory pathway follows therapeutic amputation of the hand in monkeys. J Neurosci 15:8083-8095.

Florence SL, Taub HB, Kaas JH (1998) Large-scale sprouting of cortical connections after peripheral injury in adult macaque monkeys. Science 282:1117-1121.

Graziano A, Jones EG (2006) Changes in gene expression accompanying somatosensory plasticity in adult monkeys. Soc Neurosci Abstr 32:212.8

Hill A (1999) Phantom limb pain: a review of the literature on attributes and potential mechanisms. J Pain Symptom Manage 17:125-142.

Huse E, Larbig W, Flor H, Birbaumer N (2001) The effect of opioids on phantom limb pain and cortical reorganization. Pain 90:47-55.

Jain N, Catania KC, Kaas JH (1997) Deactivation and reactivation of somatosensory cortex after dorsal spinal cord injury. Nature 386:495-498.
Jain N, Florence SL, Qi HX, Kaas JH (2000) Growth of new brainstem connections in adult monkeys with massive sensory loss. Proc Natl Acad Sci U S A 97:5546-5550.

Jain N, Qi HX, Collins CE, Kaas JH (2008) Large-scale reorganization in the somatosensory cortex and thalamus after sensory loss in macaque monkeys. J Neurosci 28:11042-11060.

Jones EG (2000) Cortical and subcortical contributions to activitydependent plasticity in primate somatosensory cortex. Annu Rev Neurosci 23:1-37.

Jones EG (2007) The Thalamus, 2 Ed. Cambridge, UK: Cambridge UP.

Jones EG, Pons TP (1998) Thalamic and brainstem contributions to large-scale plasticity of primate somatosensory cortex. Science 282:1121-1125.

Jones EG, Manger PR, Woods TM (1997) Maintenance of a somatotopic cortical map in the face of diminishing thalamocortical inputs. Proc Natl Acad Sci U S A 94:11003-11007.

Jones EG, Woods TM, Manger PR (2002) Adaptive responses of monkey somatosensory cortex to peripheral and central deafferentation. Neuroscience 111:775-797.

Kaas JH, Florence SL (2001) Reorganization of sensory and motor systems in adult mammals after injury. In: The mutable brain: dynamic and plastic features of the developing and mature brain (Kaas JH, ed), pp 165-242. Amsterdam: Harwood Academic.

Kaas JH, Qi HX, Burish MJ, Gharbawie OA, Onifer SM, Massey JM (2007) Cortical and subcortical plasticity in the brains of humans, primates, and rats after damage to sensory afferents in the dorsal columns of the spinal cord. Exp Neurol:407-416.

Knecht S, Henningsen H, Höhling C, Elbert T, Flor H, Pantev C, Taub E (1998) Plasticity of plasticity? Changes in the pattern of perceptual correlates of reorganization after amputation. Brain 121:717-724.

Knyihár-Csillik E, Rakic P, Csillik B (1987) Transganglionic degenerative atrophy in the substantia gelatinosa of the spinal cord after peripheral nerve transection in rhesus monkeys. Cell Tissue Res 247:599-604.

Knyihár-Csillik E, Rakic P, Csillik B (1989) Transneuronal degeneration in the Rolando substance of the primate spinal cord evoked by axotomy-induced transganglionic degenerative atrophy of central primary sensory terminals. Cell Tissue Res 258:515-525.

Lenz FA, Kwan HC, Martin R, Tasker R, Richardson RT, Dostrovsky JO (1994) Characteristics of somatotopic organization and spontaneous neuronal activity in the region of the thalamic principal sensory nucleus in patients with spinal cord transection. J Neurophysiol $72: 1570-1587$

Li Y, Raisman G (1995) Sprouts from cut corticospinal axons persist in the presence of astrocytic scarring in long-term lesions of the adult rat spinal cord. Exp Neurol 134:102-111.

Liss AG, Wiberg M (1997a) Loss of nerve endings in the spinal dorsal horn after a peripheral nerve injury. An anatomical study in Macaca fascicularis monkeys. Eur J Neurosci 9:2187-2192.

Liss AG, Wiberg M (1997b) Loss of primary afferent nerve terminals in the brainstem after peripheral nerve transection: an anatomical study in monkeys. Anat Embryol (Berl) 196:279-289.

Liss AG, af Ekenstam FW, Wiberg M (1996) Loss of neurons in the dorsal root ganglia after transection of a peripheral sensory nerve. An anatomical study in monkeys. Scand J Plast Reconstr Surg Hand Surg 30:1-6.

Merzenich M (1998) Long-term change of mind. Science 282:1062-1063.

Merzenich MM, Nelson RJ, Stryker MP, Cynader MS, Schoppmann A, Zook JM (1984) Somatosensory cortical map changes following digit amputation in adult monkeys. J Comp Neurol 224:591-605.

Moore CI, Stern CE, Dunbar C, Kostyk SK, Gehi A, Corkin S (2000) Referred phantom sensations and cortical reorganization after spinal cord injury in humans. Proc Natl Acad Sci USA 97:14703-14708.

Pantev C, Engelien A, Candia V, Elbert T (2001) Representational cortex in musicians. Plastic alterations in response to musical practice. Ann N Y Acad Sci 930:300-314.

Pichitpornchai C, Rawson JA, Rees S (1994) Morphology of parallel fibres in the cerebellar cortex of the rat: an experimental light and 
electron microscopic study with biocytin. J Comp Neurol 342:206-220.

Pons TP, Garraghty PE, Ommaya AK, Kaas JH, Taub E, Mishkin M (1991) Massive cortical reorganization after sensory deafferentation in adult macaques. Science 252:1857-1860.

Ramachandran VS, Rogers-Ramachandran D, Stewart M (1992) Perceptual correlates of massive cortical reorganization. Science 258:1159-1160.

Rausell E, Bickford L, Manger PR, Woods TM, Jones EG (1998) Extensive divergence and convergence in the thalamocortical projection to monkey somatosensory cortex. J Neurosci 18:4216-4232.

Recanzone GH, Merzenich MM, Jenkins WM, Grajski KA, Dinse HR (1992) Topographic reorganization of the hand representation in cortical area $3 \mathrm{~b}$ owl monkeys trained in a frequency-discrimination task. J Neurophysiol 67:1031-1056.

Sadler M, Berry M (1983) Morphometric study of the development of Purkinje cell dendritic trees in the mouse using vertex analysis. J Microsc 131:341-354.

Schroeder CE, Seto S, Arezzo JC, Garraghty PE (1995) Electrophysiological evidence for overlapping dominant and latent inputs to somatosensory cortex in squirrel monkeys. J Neurophysiol 74:722-732.

Sherrington CS, Denny-Brown D (1939) On the distribution of sensory nerve roots. In: Selected writings of Sir Charles Sherrington, pp 31-93. London: Hamish Hamilton.
Störmer S, Gerner HJ, Grüninger W, Metzmacher K, Föllinger S, Wienke C, Aldinger W, Walker N, Zimmermann M, Paeslack V (1997) Chronic pain/dysaesthesiae in spinal cord injury patients: results of a multicentre study. Spinal Cord 35:446-455.

Tan AM, Petruska JC, Mendell LM, Levine JM (2007) Sensory afferents regenerated into dorsal columns after spinal cord injury remain in a chronic pathophysiological state. Exp Neurol 206:257-268.

Ullrich DP, Woosley CN (1954) Trigeminal nerve representation in the upper head area of the postcentral gyrus of Macaca mulatta. Trans Am Neurol Assoc 13:23-28.

Wall PD (1977) The presence of ineffective synapses and the circumstances which unmask them. Philos Trans R Soc Lond B Biol Sci 278:361-372.

Weiss T, Miltner WH, Liepert J, Meissner W, Taub E (2004) Rapid functional plasticity in the primary somatomotor cortex and perceptual changes after nerve block. Eur J Neurosci 20:3413-3423.

Westrum LE, Blackstad TW (1962) An electron microscopic study of the stratum radiatum of the rat hippocampus (regio superior, CA 1) with particular emphasis on synaptology. J Comp Neurol 119:281-309.

Woods TM, Cusick CG, Pons TP, Taub E, Jones EG (2000) Progressive transneuronal changes in the brainstem and thalamus after long-term dorsal rhizotomies in adult macaque monkeys. J Neurosci 20:38843899. 